\title{
Surface waves and their influence on cone cracking in brittle materials
}

Jason McDonald and Sikhanda Satapathy

US Army Research Laboratory, RDRL-WMP-B, Aberdeen Proving Ground, MD 21005, United States

Corresponding Author:

Jason McDonald

Phone: 00-1-410-278-3707

Fax: 00-1-410-278-2460

Email: jason.r.mcdonald27.civ@ mail.mil

Abstract:

12 Prior experimental studies have shown substantial improvement in the ballistic performance of

13 ceramics due to surface treatment with thin coatings; however, no correlation with material

14 properties and bonding conditions has been found and no mechanism has yet been definitively

15 established. It is expected that the high strain rates present in a ballistic event will produce

16 surface waves and it is plausible that the interaction of these waves with surface flaws may

17 contribute significantly to initial crack growth and the onset of failure in brittle materials. In the

18 present work a Laplace-transform-based computational framework is implemented. This

19 technique can be considered complementary to other computational schemes such as finite

20 element and finite difference techniques for the study of elastic waves in solids. Extension of

21 this framework to multi-layer systems is possible and may provide insight into whether or not the

22 observed surface-treatment effect in ceramics can be attributed to wave modification.

\section{Keywords:}

24 Ceramics

25 Cracking

26 Surface waves 


\section{Introduction}

30 Surface defects and flaws likely play an important role in the impact response of brittle materials,

31 such as ceramics and glass. High speed projectile impact induces a complex evolving stress state

32 in the target. It is conceivable that the interaction of surface flaws with the evolving stress fields

33 can contribute significantly to crack initiation and the onset of failure. Prior experimental studies

34 [1-3] have shown substantial benefit of surface treatments on the ballistic efficiency of ceramics.

35 The correlation between the degree of ballistic improvement and various properties of the layer,

36 including degree of bonding with the substrate, has not definitively been established. Initial

37 attempts using finite element modeling in conjunction with accepted material models for

38 ceramics and alloys has proven unsuccessful in reproducing the experimental results [4]. One

39 possibility for this lack of success could be that surface waves of combined dilatational and

40 vertical shearing motion, often referred to as Rayleigh-type waves, are contributing significantly

41 to the onset of cracking and failure in ceramics. Waves of this type have been studied

42 extensively by seismologists over the years in both single half space and layered systems [5-7].

43 Some notable findings include: the range of existence of such modes (as a function of material

44 properties) in two-layered systems [8-10], and the dependence of their amplitude on material

45 properties and layer thickness $[11,12]$. Extension of these methods to problems of interest in

46 ballistics and impact, using modern computational techniques, may shed light on surface-

47 treatment effects in ceramics.

48 One drawback in the use of finite element and finite difference methods to model wave

49 propagation are the demands on spatial and temporal discretizations to avoid unphysical

50 numerical effects. It has been well established empirically that (in the case of monochromatic

51 waves) one needs a minimum 10 cells per wavelength (assuming linear interpolation) to prevent 
52 artifacts such as attenuation, anisotropy, dispersion, and polarization from emerging from

53 numerical errors [13]. Inhomogeneous meshes (employing either changes in element size or

54 interpolation order) can also lead to spurious oscillations [13]. As an example, a typical glass

55 with Rayleigh-wave speed $\sim 3000 \mathrm{~m} / \mathrm{s}$ subjected to a loading rate of $10 \mathrm{MHz}$ would require a

56 minimum spatial discretization of $0.03 \mathrm{~mm}$, possibly higher to achieve desired accuracy. It is

57 conceivable that ballistic impact events could produce an initial elastic loading phase of quite

58 high rate prior to the onset of inelastic response since one expects the loading rate to increase

59 with impact velocity [14]. Such high-rate loading may require a spatial discretization of

60 prohibitive computational expense and lead to very small time steps and large accumulated

61 errors in explicit methods. The Laplace-transform-based scheme implemented in the present

62 work does not suffer from this particular drawback since it does not utilize either spatial or

63 temporal discretization: the field computation at any space-time point is independent of the

64 computation at any other point. This property also makes the technique highly parallelizable.

65 The method does, however, have its own drawbacks such as the complication in treating loading

66 conditions in which the spatial and temporal dependences are not readily factorable [14].

67 The cone-crack system is one of several failure mechanisms (including radial cracks, axial

68 splitting, and comminuted zones) that are observed under dynamic indentation and ballistic

69 loading $[15,16]$. Although the details and relative importance of the various failure processes as

70 a function of loading conditions and geometry are not yet well understood, the authors are

71 currently of the belief that reducing the magnitude of tensile waves, near the surface of a brittle

72 solid, may reduce or delay the development of at least some of these failure mechanisms, thereby

73 improving ballistic performance. The goal of the present study is to investigate the wave-flaw

74 interaction near the surface of a brittle solid leading to cone cracks. The reason for focusing on 
75 the cone crack system in this initial study is that a simple model was previously presented by

76 Lawn et al [17] for the growth of cone cracks under quasi-static fields. To this end, we have

77 implemented a computational scheme to study the stress wave interaction with surface flaws.

78 Use of the Laplace transform enables analytical expressions for the various quantities of interest

79 to be obtained in the transformed space. Numerical inversion of these closed-form expressions

80 provides stress and displacement distributions as functions of space and time. The method was

81 first compared to the known analytical solutions for the surface displacement due to the idealized

82 case of a suddenly-applied point load. It was next applied to the problem of cone crack

83 extension from an existing ring-shaped flaw under dynamic frictionless loading of an elastic half

84 space by a rigid cylindrical punch. The computed cone cracks under dynamic loading conditions

85 showed significant differences compared to the case of quasi-static loading conditions as

86 calculated previously [17].

87 It is possible to extend this scheme to study the effects of indenter elasticity, interface friction,

88 and surface coatings. Including the effects of indenter properties and friction would provide

89 insight into whether the initial stage of failure under dynamic loading is strongly influenced by

90 indenter or projectile type as it is in the static case. The effects of coating properties, layer

91 thickness, and degree of bonding at the interface could also be studied to yield insights into the

92 role of surface constraints in improving ballistic response of ceramics. 


\section{2. Theoretical Background}

94 Consider a homogeneous, isotropic, linearly-elastic half space subjected to an axi-symmetric

95 surface source of compressive waves (the technique presented in the present work is not actually

96 restricted to axi-symmetric problems but the implementation in the three-dimensional case is

97 more complicated and will not be considered here [18]). Let the radial coordinate be denoted by

$98 \quad r$ and the depth coordinate by $z$. The displacement at a given point can be written in terms of

99 potentials $\phi$ and $\psi$ :

100

$$
\begin{aligned}
& u_{r}=\frac{\partial \phi}{\partial r}+\frac{\partial^{2} \psi}{\partial r \partial z} \\
& u_{z}=\frac{\partial \phi}{\partial z}-\frac{\partial^{2} \psi}{\partial r^{2}}-\frac{1}{r} \frac{\partial \psi}{\partial r} \\
& u_{\theta}=0
\end{aligned}
$$

The advantage of using potentials is that they satisfy the simple wave equations [19, 20]:

$$
\begin{gathered}
\vec{\nabla}^{2} \phi=\frac{1}{c_{d}{ }^{2}} \frac{\partial^{2} \phi}{\partial t^{2}} \quad \vec{\nabla}^{2} \psi=\frac{1}{c_{s}^{2}} \frac{\partial^{2} \psi}{\partial t^{2}} \\
c_{d}{ }^{2}=\frac{\lambda+2 \mu}{\rho} \quad c_{s}{ }^{2}=\frac{\mu}{\rho} \\
\vec{\nabla}^{2}=\frac{\partial^{2}}{\partial r^{2}}+\frac{1}{r} \frac{\partial}{\partial r}+\frac{\partial^{2}}{\partial z^{2}}
\end{gathered}
$$

In Equation (2) $\lambda$ and $\mu$ are the usual elastic constants and $\rho$ is the mass density. The approach taken in the present work is to use the Laplace transform to eliminate the time

105 derivatives in Equation (2) and obtain Helmholtz equations for both potentials:

106

$$
\begin{aligned}
& \bar{f}(r, z, s) \equiv \int_{0}^{\infty} f(r, z, t) e^{-s t} d t \\
& \vec{\nabla}^{2} \bar{\phi}=k_{d}{ }^{2} \bar{\phi} \\
& k_{d}{ }^{2}=\frac{s^{2}}{c_{d}{ }^{2}}
\end{aligned}
$$

107 Using the separation-of-variables method the general solutions can be found: 


$$
\begin{array}{cc}
\bar{\phi}(r, s)=\int_{0}^{\infty} A(k, s) e^{-\kappa_{d} z} J_{0}(k r) d k & \bar{\psi}(r, s)=\int_{0}^{\infty} B(k, s) e^{-\kappa_{s} z} J_{0}(k r) d k \\
\kappa_{d}{ }^{2}=k_{d}{ }^{2}+k^{2} & \kappa_{s}{ }^{2}=k_{s}{ }^{2}+k^{2}
\end{array}
$$

109 In Equation (4) $J_{0}$ is the zeroth-order Bessel function of the first kind. Inserting the expressions

110 in Equation (4) into the expressions in Equation (1) gives the general expressions for the

111 displacements:

112

$$
\begin{aligned}
& \bar{u}_{r}=\int_{0}^{\infty}\left(-A(k) e^{-\kappa_{d} z}+\kappa_{s} B(k) e^{-\kappa_{s} z}\right) k J_{1}(k r) d k \\
& \bar{u}_{z}=\int_{0}^{\infty}\left(-\kappa_{d} A(k) e^{-\kappa_{d} z}+k^{2} B(k) e^{-\kappa_{s} z}\right) J_{0}(k r) d k \\
& \bar{u}_{\theta}=0
\end{aligned}
$$

113 General expressions for the stresses can be determined using the following relations:

114

$$
\begin{aligned}
& \bar{\sigma}_{r}=\lambda\left(\frac{\partial \bar{u}_{r}}{\partial r}+\frac{\bar{u}_{r}}{r}+\frac{\partial \bar{u}_{z}}{\partial z}\right)+2 \mu \frac{\partial \bar{u}_{r}}{\partial r} \\
& \bar{\sigma}_{z}=\lambda\left(\frac{\partial \bar{u}_{r}}{\partial r}+\frac{\bar{u}_{r}}{r}+\frac{\partial \bar{u}_{z}}{\partial z}\right)+2 \mu \frac{\partial \bar{u}_{z}}{\partial z} \\
& \bar{\sigma}_{\theta}=\lambda\left(\frac{\partial \bar{u}_{r}}{\partial r}+\frac{\bar{u}_{r}}{r}+\frac{\partial \bar{u}_{z}}{\partial z}\right)+2 \mu \frac{\bar{u}_{r}}{r} \\
& \bar{\sigma}_{r z}=\mu\left(\frac{\partial \bar{u}_{r}}{\partial r}+\frac{\partial \bar{u}_{z}}{\partial z}\right)
\end{aligned}
$$

115 Inserting Equation (5) into Equation (6) gives: 


$$
\begin{aligned}
\bar{\sigma}_{r}= & \int_{0}^{\infty}\left(\left(\lambda k_{d}{ }^{2}-2 \mu k^{2}\right) A(k) e^{-\kappa_{d} z}+2 \mu k^{2} \kappa_{s} B(k) e^{-\kappa_{s} z}\right) J_{0}(k r) d k \\
& +2 \mu \int_{0}^{\infty} k^{2}\left(A(k) e^{-\kappa_{d} z}-\kappa_{s} B(k) e^{-\kappa_{s} z}\right) \frac{J_{1}(k r)}{k r} d k \\
\bar{\sigma}_{z}= & \int_{0}^{\infty}\left(\left(\lambda k_{d}{ }^{2}+2 \mu \kappa_{d}{ }^{2}\right) A(k) e^{-\kappa_{d} z}-2 \mu k^{2} \kappa_{s} B(k) e^{-\kappa_{s} z}\right) J_{0}(k r) d k \\
\bar{\sigma}_{\theta}= & \int_{0}^{\infty} \lambda k_{d}{ }^{2} A(k) e^{-\kappa_{d} z} J_{0}(k r) d k+2 \mu \int_{0}^{\infty} k^{2}\left(-A(k) e^{-\kappa_{d} z}+\kappa_{s} B(k) e^{-\kappa_{s} z}\right) \frac{J_{1}(k r)}{k r} d k \\
\bar{\sigma}_{r z}= & \int_{0}^{\infty} \mu k\left(2 \kappa_{d} A(k) e^{-\kappa_{d} z}-\left(\kappa_{s}{ }^{2}+k^{2}\right) B(k) e^{-\kappa_{s} z}\right) J_{1}(k r) d k
\end{aligned}
$$

117 The coefficients $A$ and $B$ are determined from the boundary conditions:

$$
\begin{aligned}
& \bar{\sigma}_{z}(r, 0, s)=-F(r) \bar{G}(s) \\
& \bar{\sigma}_{r z}(r, 0, s)=0
\end{aligned}
$$

119 In the present work we only consider loading conditions in which the spatial and temporal

120 responses can be separated. The technique for treating more general cases has been presented by

121 Tsai [14] and may be incorporated in future work. Expressions for the coefficients for various

122 cases are given in the Appendix.

\section{3. Numerical Solution Method}

125 Numerical evaluation of the expressions in Equations (5) and (7) presents several difficulties

126 which must be addressed to obtain an accurate and stable computational scheme. One

127 complication involves the oscillatory nature of the Bessel functions appearing in the integrands;

128 if not properly addressed this behavior will cause prohibitively slow convergence in any

129 numerical quadrature scheme. The solution is to subdivide the integral into a summation of sub 130 integrals between the zeros of the Bessel function $J_{n}(x)$ : 


$$
\begin{aligned}
& \int_{0}^{\infty} f(x) J_{n}(x) d x=\sum_{j=0}^{\infty} \int_{x_{j}^{(n)}}^{x_{j+1}^{(n)}} f(x) J_{n}(x) d x \\
& x_{0}^{(n)}=0, J_{n}\left(x_{i=1,2, \ldots}^{(n)}\right)=0
\end{aligned}
$$

132 The convergence of the resulting series is then accelerated by applying the epsilon algorithm of

133 Wynn [21]. The algorithm transforms, using a non-linear interation, the sequence of partial sums

134 of the infinite series in Equation (9), into a new sequence (the so-called diagonal Padé

135 approximants) having the same limit as the original sequence but typically better convergence 136 properties. In the present work we used the Quadpack [22] routines QAGP, for evaluating the 137 sub-integrals in Equation (9), and QEXT for the epsilon algorithm. For the QAGP routine we 138 chose a 61-point Kronrad integration rule, as described in chapter 2 of reference [22], to ensure

139 high accuracy in the evaluation of the sub-integrals. Both routines require a user-specified

140 relative tolerance to determine the stopping criteria. For the present work we used a relative 141 tolerance of $10^{-8}$.

142 A second difficulty in evaluating the integrals involves the branch points associated with the 143 dilatational $(\mathrm{P})$ and shear $(\mathrm{S})$ waves:

$$
\begin{aligned}
& k_{b}^{(d)}=\frac{\operatorname{Im}(s)}{c_{d}} \\
& k_{b}^{(s)}=\frac{\operatorname{Im}(s)}{c_{s}}
\end{aligned}
$$

145 and the pseudo pole associated with the Rayleigh $(\mathrm{R})$ wave $k_{p}^{(r)}$, which is given by the real root

146 of the Rayleigh function:

$$
\left(2 k_{p}^{(r)^{2}}+k_{s}^{2}\right)^{2}-4 \kappa_{d} \kappa_{s} k_{p}^{(r)^{2}}=0
$$

148 In the present work Equation (11) is solved by iteration using the secant method described in 149 [23]. Special care must be taken to isolate these points for special treatment in order to ensure 
150 accuracy and stability of the method [20]. For the routine QAGP, used in the present work, the

151 user can specify a set of internal points for further subdivision of the original interval (between

152 successive zeros of the Bessel function). This allows problem points (where changes of slope or

153 large gradients occur) to be identified for use as endpoints. The two branch points and the

154 pseudo pole must all be specified as internal points; in addition, for the pseudo pole it was found

155 necessary to specify four extra points on either side of it in order to achieve sufficient accuracy

156 to capture its effect.

157 In order to obtain the final time-domain solutions an inverse Laplace transform routine must be

158 used. The routine chosen for the present study was a revision of routine INVLTF which is

159 described in detail in [24]. It utilizes a Fourier-series representation of the Bromwich integral

160 and the QD algorithm (which calculates successive Padé approximations in the form of

161 continued fractions) to accelerate the convergence of the resulting series. The formally infinite

162 series, which results from discretization of the Bromwich integral, is truncated once a user-

163 specified relative accuracy is obtained. In the present work we used a relative accuracy of $10^{-3}$

164 and default values for all other parameters described in [24].

165

166 4. Results

167 4.1 Verification of the implemention

168 In order to verify the numerical solution method we utilized the result of Pekeris [25] for the case

169 of a suddenly-applied compressive point load located at the origin (Figure 1). He obtained

170 closed-form solutions for both the vertical and horizontal displacements at the surface in terms of

171 rational functions and elliptic integrals.

172 


$$
F(r)=F_{0} \delta(r) / 2 \pi r=\lim _{a \rightarrow 0}\left(\left(F_{0} / \pi a^{2}\right) \exp \left(-r^{2} / a^{2}\right)\right)
$$

173
Figure 1: A compressive point load of strength $F_{0}$ is applied to the surface of an elastic half space. The spatial dependence can also be viewed as the limiting case of a Gaussian pulse. The temporal behavior of the load is given by the Heavyside function

$$
(H(t<0)=0, H(t>0)=1) .
$$

Since the analytical solution contains singularities it is impossible for any numerical technique to exactly reproduce it, therefore, the verification method employed was to show that the numerical solution obtained for a suddenly-applied load, with a Gaussian spatial dependence, approaches the closed-form solution of Pekeris in the far field region $(r>>a)$. The loading function assumed is given by:

$$
\begin{aligned}
& \sigma_{z}(r, 0, t)=-\frac{F_{0}}{\pi a^{2}} \exp \left(-r^{2} / a^{2}\right) H(t) \\
& \sigma_{r z}(r, 0, t)=0
\end{aligned}
$$

A value of $a=1 \mathrm{~mm}$ was chosen since projectiles in ballistics applications typically have lateral dimensions measured in $\mathrm{mm}$. Computed results for the vertical and horizontal surface displacements, for three values of the relative field point $(r / a=10,100$, and 1000), are shown in figures 2 and 3 respectively, along with the closed-form solutions for a point source. The figures demonstrate how the computed solutions approach the point-source results for $r>a$. It was found that as the value of $(r / a)$ becomes large the convergence of the summation resulting 
191 from the discretization of the integrals over $k$ becomes much slower. The origin of this behavior

192 is not well understood but it may be due to the difficulty of the numerical method to capture the

193 behavior of the solution in the vicinity of the pseudo discontinuity/singularity associated with the

194 Rayleigh-wave arrival. Fortunately this behavior is not a serious issue for the application of

195 interest in the present work since we want to model the material response in the near and

196 intermediate field regions $(r / a<50)$ where this convergence issue does not manifest itself.

197

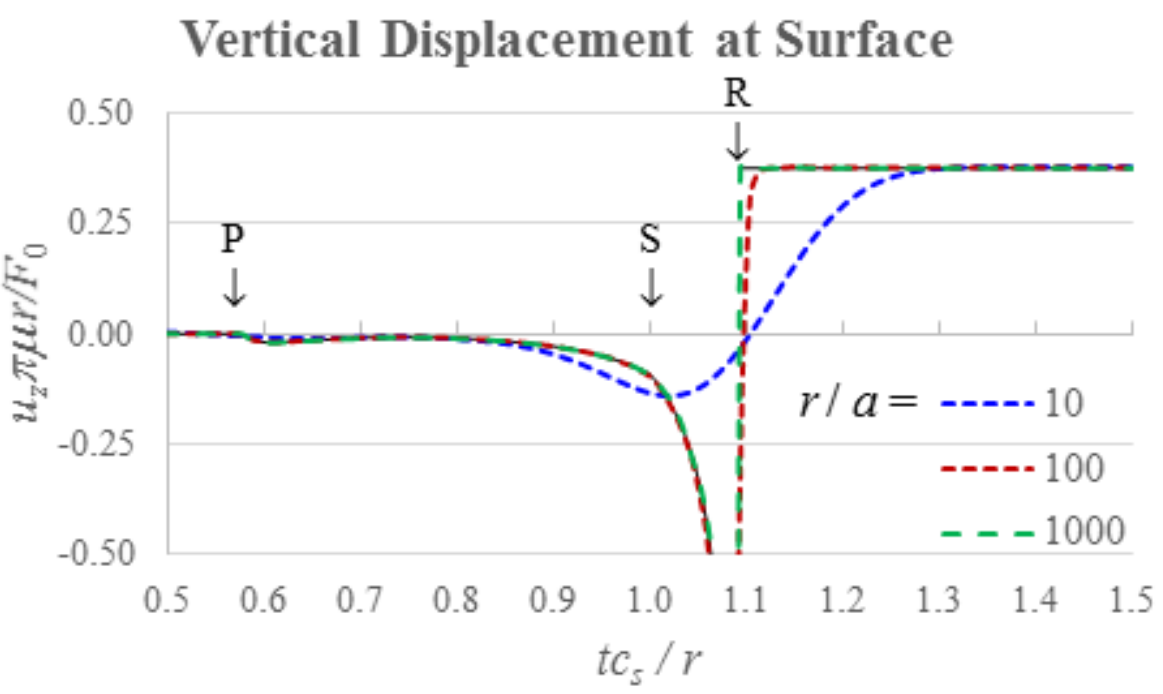

198

199

200

201

202

203

204

205

206

207

208
Figure 2: Comparison of results computed using the approach outlined in the present work versus the closed-form expressions (black solid line) obtained by Pekeris [25]. The load was taken to be a compressive Gaussian pulse of strength $F_{0} \quad\left(F(r)=F_{0} / \pi a^{2} \exp \left(-r^{2} / a^{2}\right)\right)$, with Heavyside temporal response $(G(t)=H(t))$. It was assumed that $\lambda=\mu, c_{d}=5700 \mathrm{~m} / \mathrm{s}$, and $a=1 \mathrm{~mm}$. The results in this figure are for the normalized vertical displacement $\left(=u_{z} \pi \mu r / F_{0}\right)$ versus normalized time $\left(=t c_{s} / r\right)$, at location $r$ on the surface $(z=0)$. The result has a singularity at the arrival of the Rayleigh wave $\left(t c_{s} / r \approx 1.088\right)$. A small bump is observed at the arrival of the $\mathrm{P}$ wave $\left(t c_{s} / r \approx 0.577\right)$ and a sudden change in slope at the arrival of the $\mathrm{S}$ wave $\left(t c_{s} / r=1.0\right)$. 


\section{Horizontal Displacement at Surface}

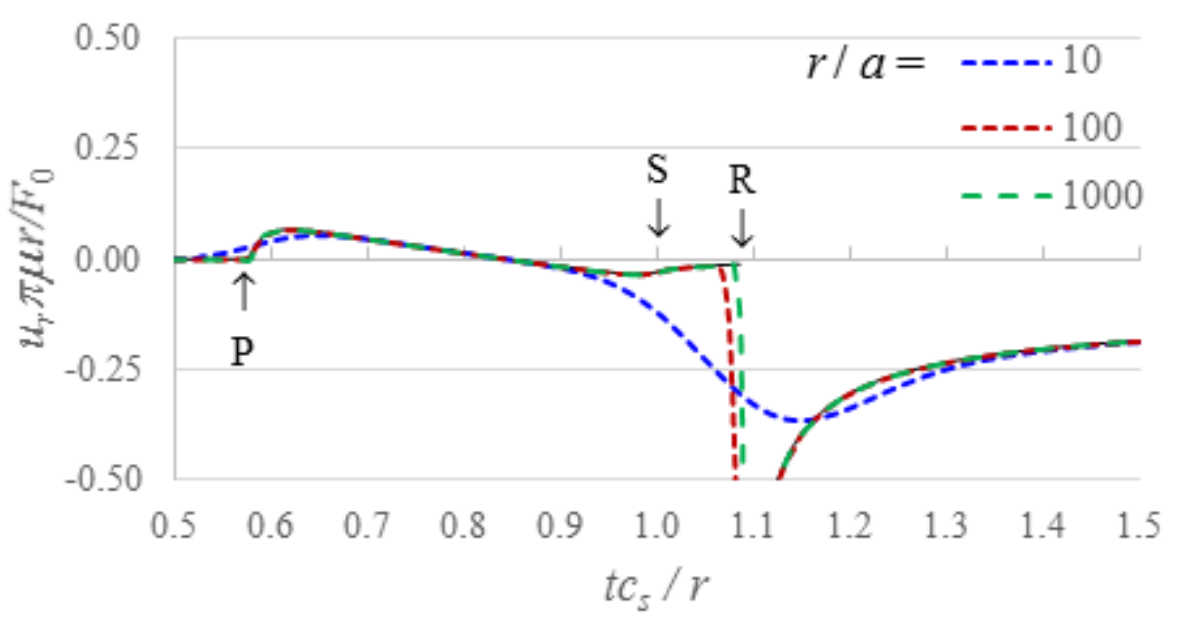

Figure 3: Comparison of results computed using the approach outlined in the present work versus the closed-form expressions obtained by Pekeris [25]. The load was taken to be a compressive Gaussian pulse of strength $F_{0} \quad\left(F(r)=F_{0} / \pi a^{2} \exp \left(-r^{2} / a^{2}\right)\right)$, with

\section{4.2. Cylindrical punch indenter}

223 In the literature the point-source solution is often employed, as the kernel in a convolution

224 integral, to construct solutions for arbitrary source distributions. For our purposes this is not a

225 practical approach because of the difficulty in computing the point-source solution. It is far more 226 efficient to find the numerical solution to the system defined in (5), (7) and (8) for the particular 227 source of interest. The sphere and the cylinder are often employed in dynamic indentation and 
228 ballistic experiments. For the method employed in the present work it is far simpler to consider

229 the cylindrical flat-punch (Figure 4) because the radius of the contact circle stays fixed as the

230 load is increased which allows for a factorization of spatial and temporal dependences consistent

231 with equation (8). The procedure for treating the sphere has been described by Tsai [14] but it is

232 considerably more difficult and will not be considered in the present work. The actual loading

233 conditions for an impact event have only been derived for the quasi-static case in which wave

234 effects can be neglected (the Hertzian theory). Tsai [14] presented an approach for deriving the

235 first correction term to Hertzian theory for a sphere but the mathematics is quite involved. In the

236 present work we will use an ansatz for the loading condition on an elastic half space by a

237 cylindrical indenter in the dynamic regime. We assume that the cylinder is rigid (infinite elastic

238 moduli) and that there is no friction between the contacting surfaces. We will also neglect

239 inelastic and dissipative effects. In the case of static loading the distribution of pressure on the

240 surface is given by the well-known Hertzian solution [26]:

$$
F(r)=\left\{\begin{array}{cc}
F_{0} /\left(2 \pi a\left(a^{2}-r^{2}\right)^{1 / 2}\right) & r<a \\
0 & r>a
\end{array}\right.
$$

242 We will assume that we can use the Hertzian result in Equation (13) for the spatial dependence

243 of our loading condition and that the time dependence is given by a simple ramp function:

$$
G(t)=\left\{\begin{array}{cc}
0 & t \leq 0 \\
t / \tau & 0<t<\tau \\
1 & t \geq \tau
\end{array}\right.
$$

245 It is unlikely that this loading condition represents the actual dynamic loading condition that

246 occurs during impact but it is a convenient way of producing elastic waves whose effects can be 247 studied. 


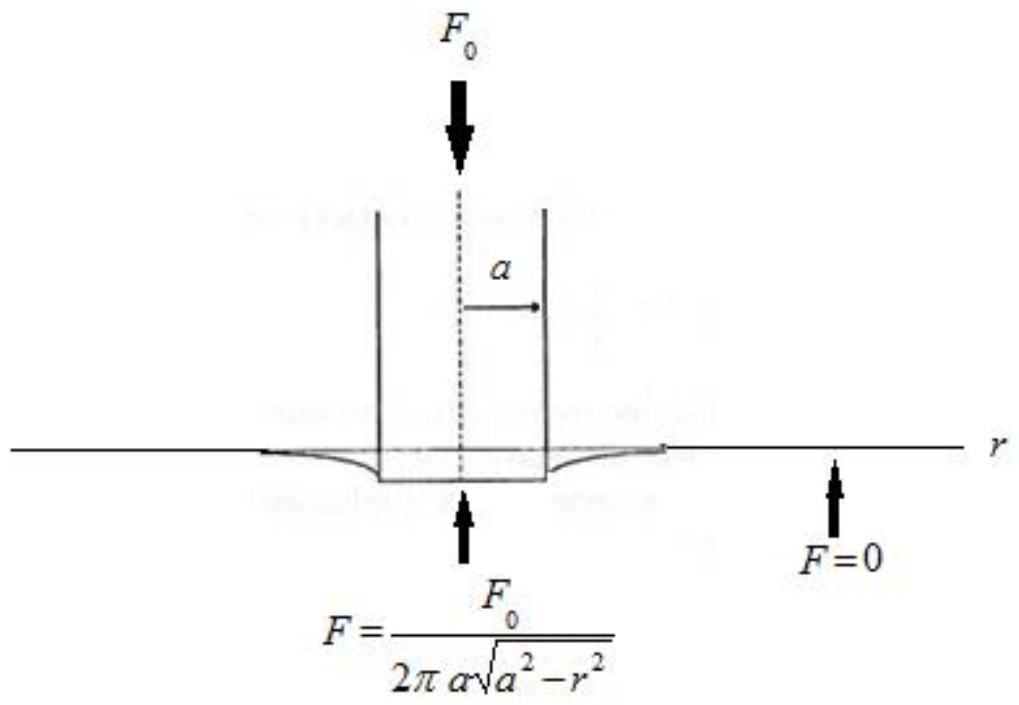

248

Figure 4: Cylindrical flat-punch indenter of radius $a$ and total load strength $F_{0}$. The spatial distribution of the normal stress on the surface is given by: $\left(F(r<a)=\left(F_{0} / 2 \pi a\right) /\left(a^{2}-r^{2}\right)^{1 / 2}, F(r>a)=0\right)$. In the case of static loading the radial and hoop stresses on the surface, outside the contact circle, are given by: $\sigma_{r}(r>a, z=0)=F_{0}(1-2 v) / 2 \pi r^{2}$ and $\sigma_{\theta}(r>a, z=0)=-F_{0}(1-2 v) / 2 \pi r^{2}$ respectively.

Figures 5 and 6 show the vertical and horizontal displacement of the surface at a radial value $r=5 a$ for various values of the ramp time $\tau$. The limit $\tau \rightarrow 0$ corresponds to the extreme dynamic case (suddenly-applied load) while in the limit $\tau \rightarrow \infty$ the material should instantaneously assume the static solution at any given load value. It is apparent from the figures that the solutions approach the static-solution values in the limit $t c_{s} / a>>1$ as would be expected. The solutions also develop sharper features as $\tau$ decreases. 


\section{Vertical Displacement}

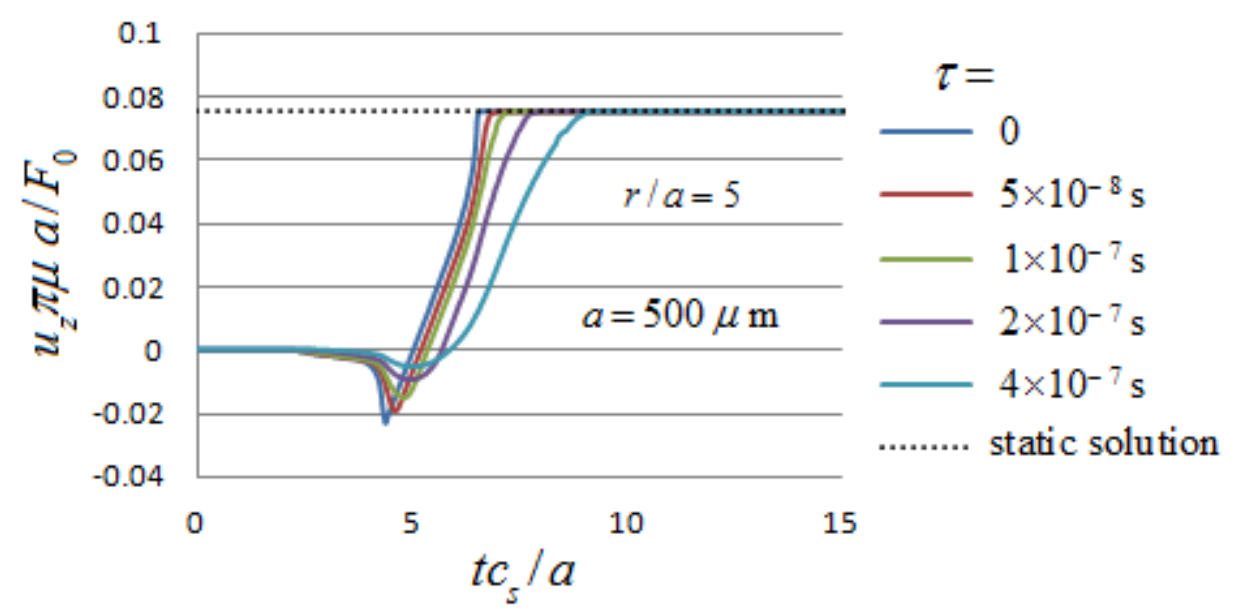

Figure 5: Computed normalized vertical displacement $\left(=u_{z} \pi \mu a / F_{0}\right)$ versus normalized time $\left(=t c_{s} / a\right)$, at location $r / a=5$ on the surface $(z=0)$. The load was taken to be a cylindrical-punch indenter of strength $F_{0}$ and radius $a=500 \mu \mathrm{m}$

Horizontal Displacement

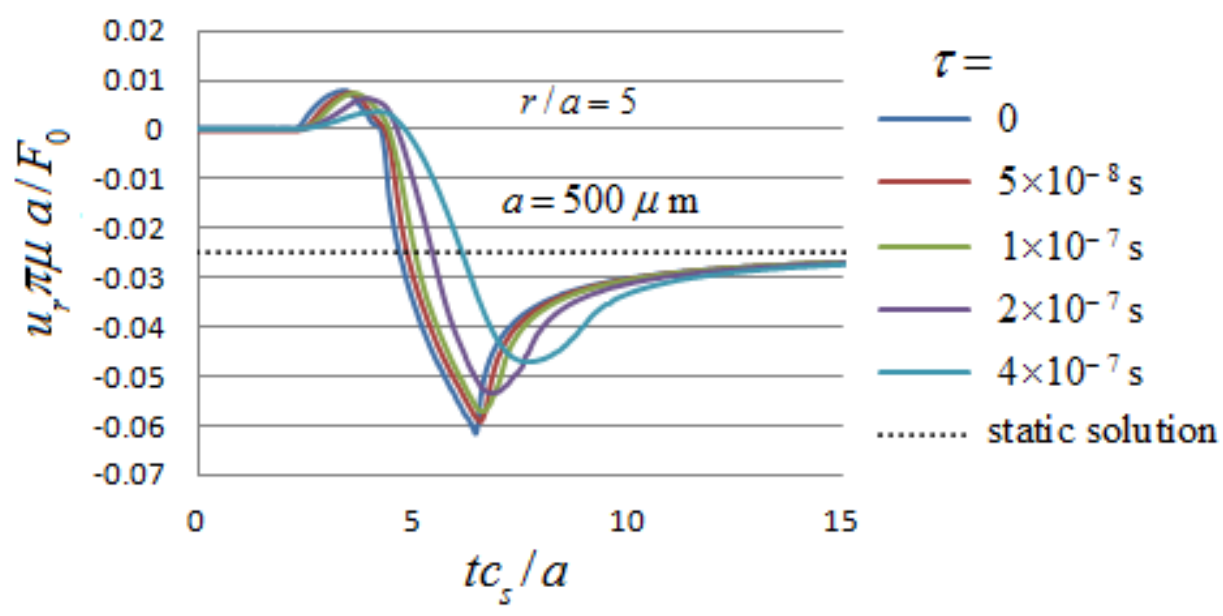


Figure 6: Computed normalized horizontal displacement $\left(=u_{r} \pi \mu a / F_{0}\right)$ versus normalized time $\left(=t c_{s} / a\right)$, at location $r / a=5$ on the surface $(z=0)$. The load was taken to be a cylindrical-punch indenter of strength $F_{0}$ and radius $a=500 \mu \mathrm{m}$ $\left(F(r<a)=\left(F_{0} / 2 \pi a\right) /\left(a^{2}-r^{2}\right)^{1 / 2}, F(r>a)=0\right)$. A ramped temporal response

281 Figures 7 and 8 show the largest $r$-z plane principal stress $\sigma_{1}$ and the principal stress in the 282 azimuthal direction $\sigma_{2}$ for the same simulations. It is clear that the in-plane stress develops a 283 very significant tensile peak as $\tau$ decreases which appears to sharpen into a singularity in the 284 limit $\tau \rightarrow 0$. This tensile peak can be very important with regard to cone cracking under 285 dynamic loading as discussed in the next section. It is also interesting to note that the hoop stress 286 develops a tensile peak in the dynamic regime whereas in the static regime it is compressive. 287 This would tend to imply that it is possible to open radial cracks in the dynamic case but not in 288 the static one. This is in agreement with dynamic indention experiments which show that below 289 a certain impact velocity only the cone-crack system appears while above it the radial-crack 290 system starts to appear [16, 27]. 
Maximum Principal Stress in $r$-z Plane

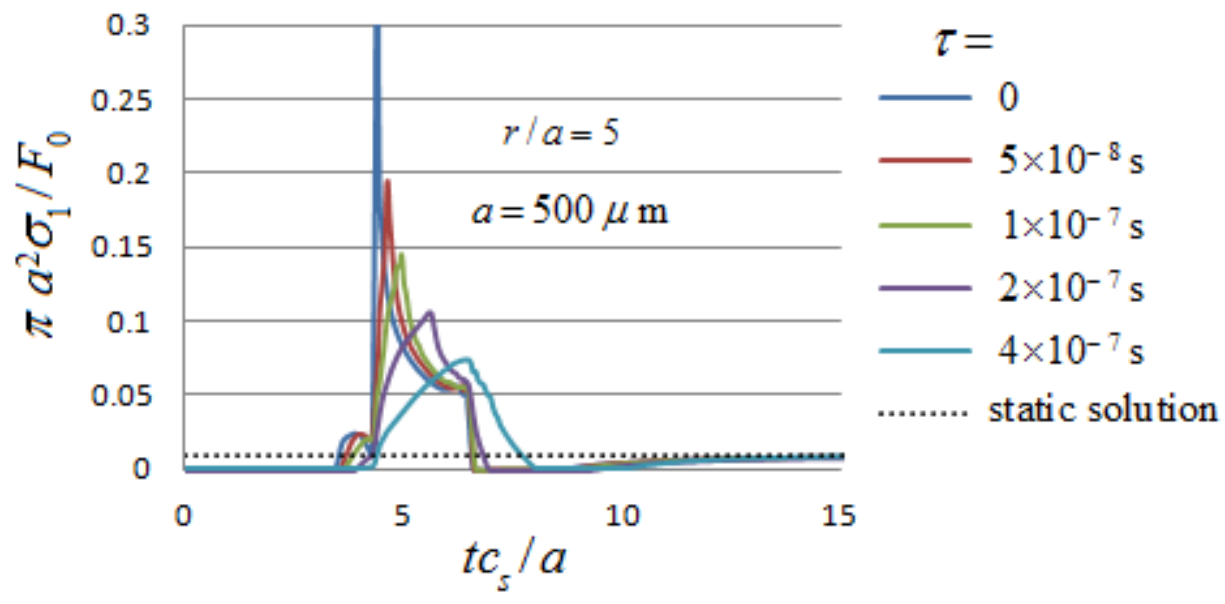

Figure 7: Computed normalized in-plane tensile stress $\left(=\pi a^{2} \sigma_{1} / F_{0}\right)$ versus normalized time $\left(=t c_{s} / a\right)$, at location $r / a=5$ on the surface $(z=0)$. The load was taken to be a cylindrical-punch indenter of strength $F_{0}$ and radius $a=500 \mu \mathrm{m}$ : $\left(F(r<a)=\left(F_{0} / 2 \pi a\right) /\left(a^{2}-r^{2}\right)^{1 / 2}, F(r>a)=0\right)$. A ramped temporal response $(G(t<0)=0, G(0<t<\tau)=t / \tau, G(t>\tau)=1)$ was assumed. The features become less sharp as the ramp time $\tau$ is increased. The case $\tau=0$ corresponds to the Heavyside function $(G(t)=H(t))$. It is clear that for sufficiently small $\tau$ (dynamic loading) there is a substantial increase in the tensile stress as compared to static loading. 


\section{Principal Stress in Azimuthal Direction}

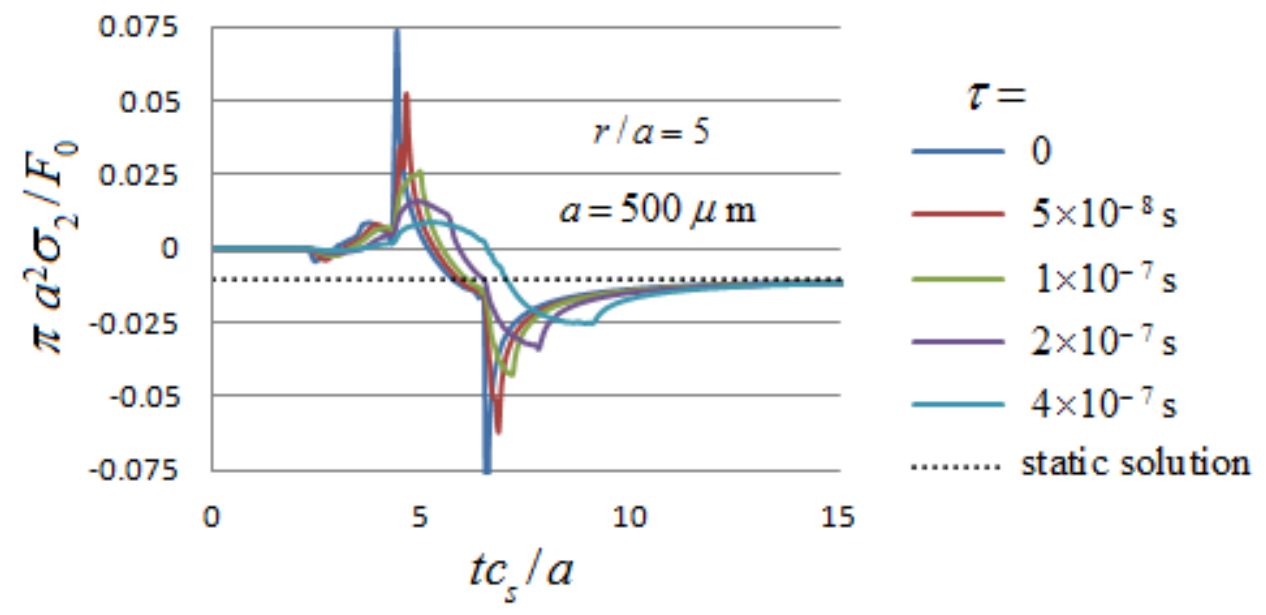

302

312 It follows from dimensional analysis that the shapes of the computed curves are determined by

313 the value of the dimensionless number $c_{d} \tau / a$ with $c_{d} \tau / a>>1$ corresponding to quasi-static

314 behavior and $c_{d} \tau / a<<1$ to highly-dynamic behavior. The results of the present work indicate

315 that even for $c_{d} \tau / a \sim 1$ the behavior is already fairly dynamic in nature.

316 It was mentioned previously that in the dynamic case the peak tensile stress becomes much

317 larger than in the static case. In addition, the peak tensile stress in the dynamic case falls off 318 much less rapidly with distance from the source than in the static case. Figure 9 shows that for 
$319 c_{d} \tau / a=1.14$ the peak tensile stress falls off approximately as $(r / a)^{-0.5}$ in contrast to $(r / a)^{-2}$

320 for the static case $c_{d} \tau / a \rightarrow \infty$. One can also observe the separation of the different components

321 of the net waveform as the observation point moves further from the source (a smaller hump

322 arrives at earlier times followed by a larger spike). This is due to the different components

323 (associated with the P, S, and R modes) having different velocities. The portion of the net

324 waveform associated with the $\mathrm{P}$ and $\mathrm{S}$ modes falls off as $(r / a)^{-2}$ and becomes broader as it

325 propagates; in contrast, the portion associated with the $\mathrm{R}$ mode displays $(r / a)^{-0.5}$ asymptotic

326 dependence. These results for the asymptotic behavior of the various components is well-known

327 and it is reassuring that the present approach reproduces them. In the next section it will be

328 shown that these difference in asymptotic behavior lead to profound differences in the cone

329 cracking behavior under dynamic and quasi-static loading.

Maximum Principal Stress in $r$-z Plane

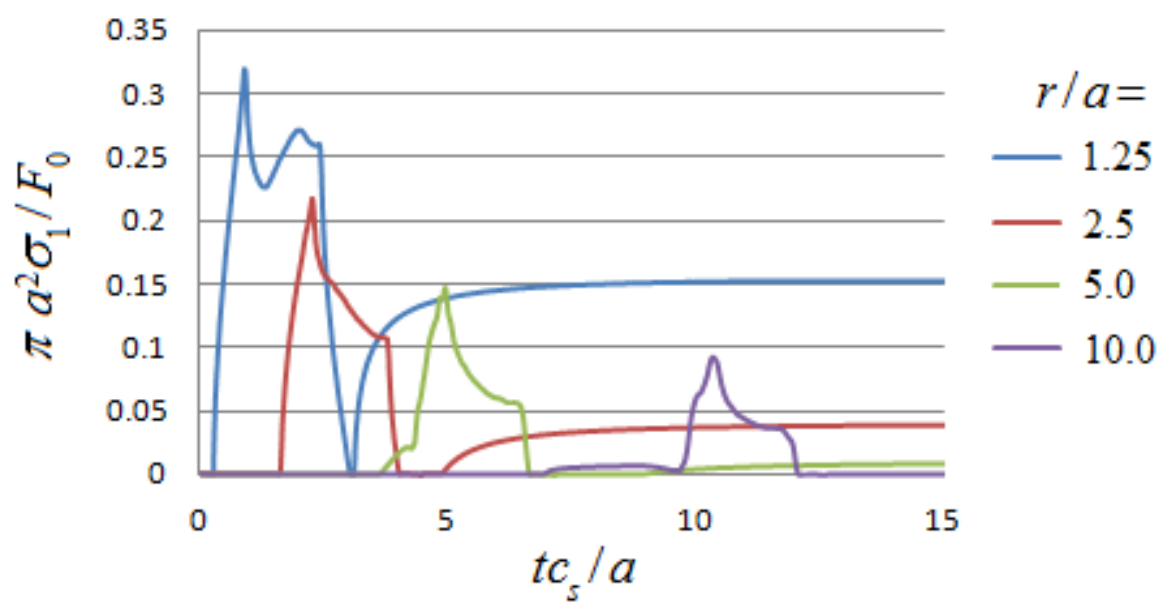

$331 \quad$ Figure 9: Computed normalized in-plane tensile stress $\left(=\pi a^{2} \sigma_{1} / F_{0}\right)$ versus normalized 332 time $\left(=t c_{s} / a\right)$, at various locations $(r / a)$ on the surface $(z=0)$. The load was taken to 333 be a cylindrical-punch indenter of strength $F_{0}$ and radius $a=500 \mu \mathrm{m}$ :

$334\left(F(r<a)=\left(F_{0} / 2 \pi a\right) /\left(a^{2}-r^{2}\right)^{1 / 2}, F(r>a)=0\right)$. The temporal response was assumed 
to be ramped $(G(t<0)=0, G(0<t<\tau)=t / \tau, G(t>\tau)=1)$ with ramp length $\tau=10^{-7} \mathrm{sec}$. The dilatational wave speed was assumed to be $c_{d}=5700 \mathrm{~m} / \mathrm{s}$ and the Poisson ratio $v=0.25$. The characteristic dimensionless number in this case is

\subsection{Cone cracks: static versus dynamic loading}

342 cracks formed in brittle materials under dynamic loading by an indenter. Studies under quasi-

343 static conditions are numerous and date all the way back to Hertz's investigations in the 1880's

344 [28]. A detailed computational study of quasi-static cone cracking in soda-lime glass due to a

345 spherical indenter was presented by Lawn et al [17]. The goal in the present study was to

346 perform a similar calculation under dynamic loading conditions so that the predicted cracks can

347 be compared to those in the quasi-static case. The basic idea is illustrated in Figure 10.

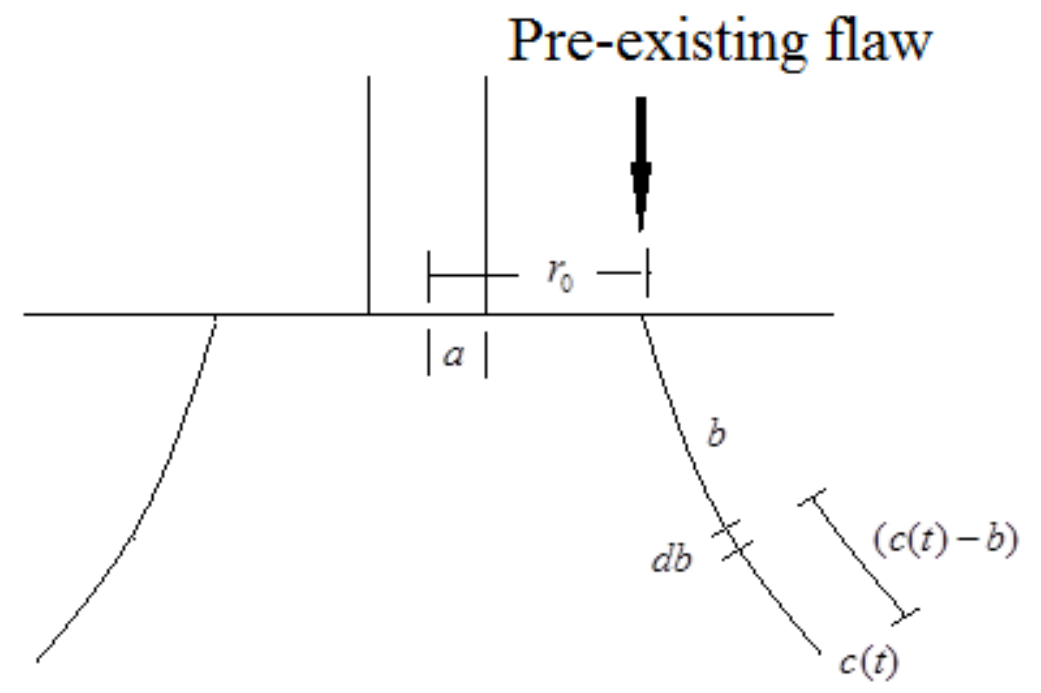


cylindrical flat-punch of radius $a$. The rate of growth of the crack length $c$ is determined by a function of the stress intensity factor $K_{I}$ as expressed in equation (15). The intensity factor is calculated from an integral of the local tensile stress weighted by a

356 A pre-existing ring-shaped flaw is assumed to exist on the surface of a half space. A rigid 357 cylindrical indenter, of radius $a$, is applied (without friction) to the surface with a given force $358 \quad F_{0}$ and a ramp time $\tau$. The crack extends due to the tensile stress acting along the existing 359 crack path (mode I) and is assumed to follow the unit eigenvector of the principal stress $\sigma_{3}$ 360 (perpendicular to $\sigma_{1}$ ) at the existing crack tip. The growth rate of the crack is assumed to be 361 given by some function, experimentally determined, of the stress intensity factor $K_{I}$ :

$$
\frac{d c}{d t}=\mathrm{v}\left(K_{I}\right)
$$

363 At time $t$ Equation (15) is used to compute an increment of crack extension $\Delta c(t)=\mathrm{v}\left(K_{I}(t)\right) \Delta t$

364 which is added to the existing crack path. The direction of crack extension is assumed to be 365 given by the unit eigenvector of the principal stress $\sigma_{3}$ (perpendicular to $\sigma_{1}$ ) at the crack tip.

366 The value of $K_{I}(t)$ is determined using an approximation similar to that employed in [17] for

367 the case of static fields. In that work the intensity factor was calculated by integrating, along the 368 existing crack path, the component of the tensile stress normal to the crack surface weighted by 369 the appropriate Green's function. One approximation in the approach is that the Green's

370 function used is for a planar crack in the plane-strain approximation rather than a cone crack

371 which possesses curvature. A second approximation is that the crack is assumed to grow in the 372 stress field of the uncracked solid. In the present case we approximate the effects of wave 373 loading by using the retarded stress to account for the fact that the stress acting at a point along 
374 the crack path will not produce a positive contribution to the intensity factor until the arrival of

375 the corresponding Rayleigh wave at the crack tip [29, 30].:

$$
K_{I}(t)=2 \sqrt{\frac{c(t)}{\pi}} \int_{0}^{c(t)} \frac{\sigma\left(b, t-(c(t)-b) / c_{R}\right)}{\sqrt{c(t)^{2}-b^{2}}} d b
$$

377 In equation (16) $c(t)-b$ is the length of existing crack path between point $b$ and the crack tip

$378 c(t)$ (see Figure 10), and $c_{R}$ is the Rayleigh-wave speed. In the present work we are also

379 neglecting the reduction in intensity factor during rapid crack growth [29, 30]. The reason for

380 not including this effect in this initial study was the complication in designing a stable non-linear

381 iteration due to the exponential behavior of the $\mathrm{v}\left(K_{I}\right)$ curve when changing between the quasi-

382 static and dynamic growth regimes (see figure 12). We are continuing to investigate this and

383 hope to include this effect in future work. In [17] a curve for $\mathrm{v}\left(K_{I}\right)$, based on experimental

384 studies of soda-lime glass was presented and is shown in figure 12. We will use this curve for

385 the present study.

386

\section{Crack Speed versus Intensity Factor}

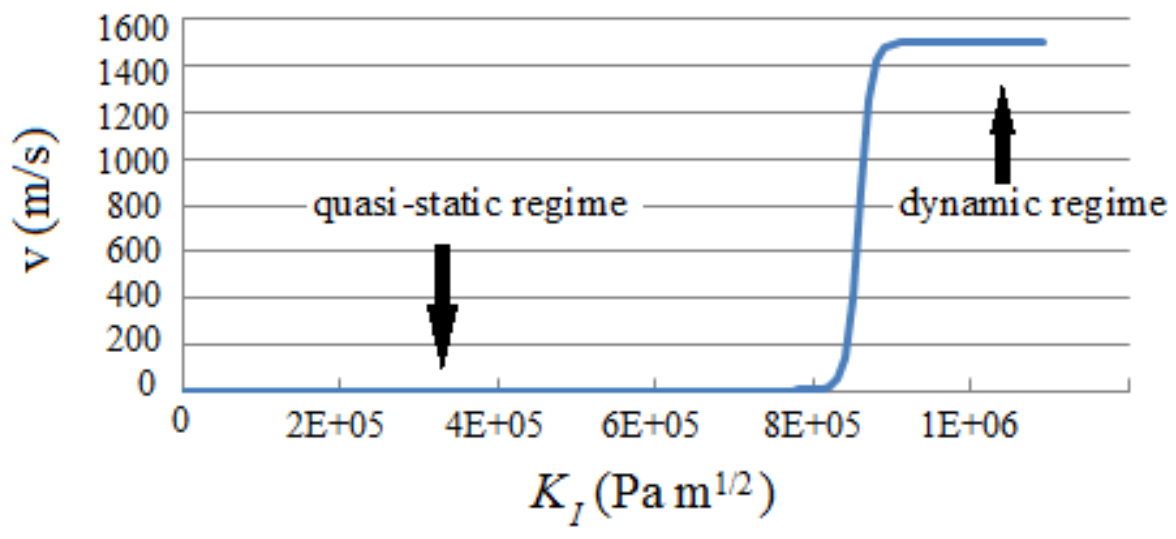


Figure 12: Crack speed versus intensity factor function $\mathrm{v}\left(K_{I}\right)$ used in the calculations. It is based on experimental work for soda-lime glass [17].

392 Although it has been pointed out that this approach does not quantitatively predict the correct

393 crack angle $[17,31]$ it does seem to reproduce much of the qualitative behavior observed in 394 experiment.

395 A comparison of the predicted crack angles for both the quasi-static $c_{d} \tau / a=1.14 \times 10^{6}$ and 396 dynamic $c_{d} \tau / a=1.14$ cases are given in figures 13,14 and 15 .

\section{Cone Cracks for Various Flaw Locations (Quasi-static Loading)}

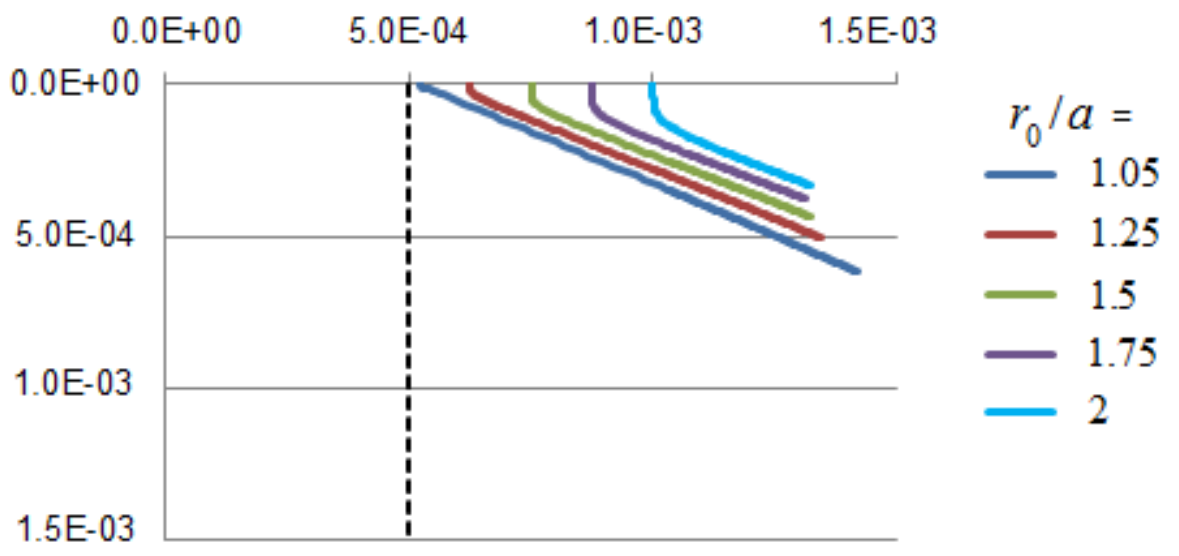

Figure 13: Cone cracks created from existing flaws, at various distances from the edge of a cylindrical flat-punch indenter, by a quasi-static load of strength $F_{0}=20 \mathrm{~N}$. The indenter radius was taken to be $a=500 \mu \mathrm{m}$, the ramp time $\tau=0.1 \mathrm{~s}$, and the dilatational wave speed $c_{d}=5700 \mathrm{~m} / \mathrm{s} \quad\left(c_{d} \tau / a=1.14 \times 10^{6}\right)$. The Poisson ratio was assumed to be $v=0.21$ which is typical of soda-lime glass. 


\section{Cone Cracks for Various Flaw Locations} (Dynamic Loading)

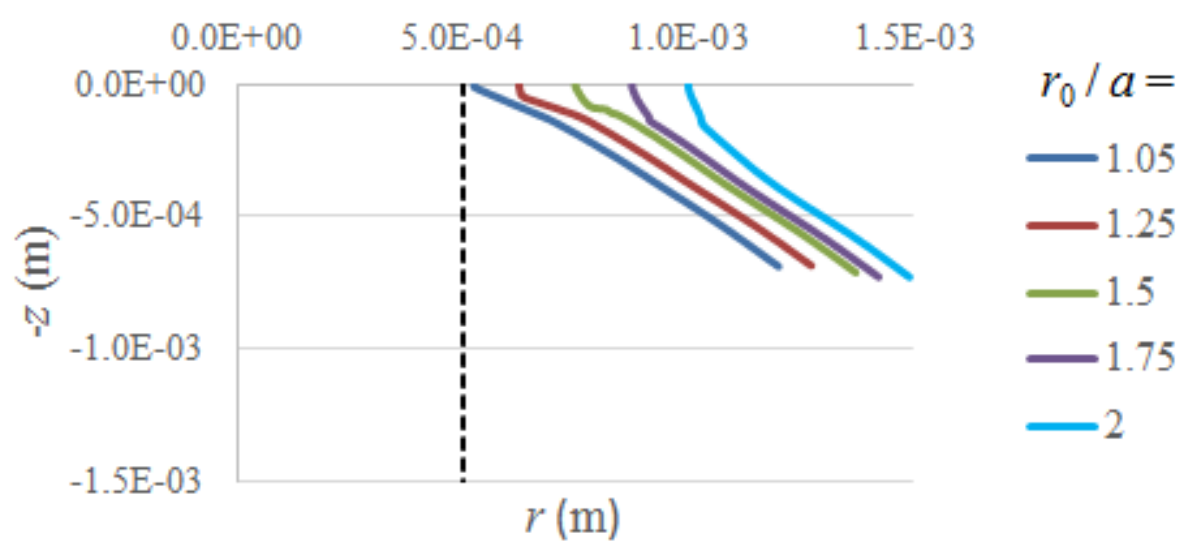

405

406

407

408

409

410

411

Figure 14: Cone cracks created from existing flaws, at various distances from the edge of a cylindrical flat-punch indenter, by a dynamic load of strength $F_{0}=20 \mathrm{~N}$. The indenter radius was taken to be $a=500 \mu \mathrm{m}$, the ramp time $\tau=10^{-7} \mathrm{~s}$, and the dilatational wave speed $c_{d}=5700 \mathrm{~m} / \mathrm{s}\left(c_{d} \tau / a=1.14\right)$. The Poisson ratio was assumed to be $v=0.21$ which is typical of soda-lime glass.

Cone Crack Shapes (Static vs Dynamic)

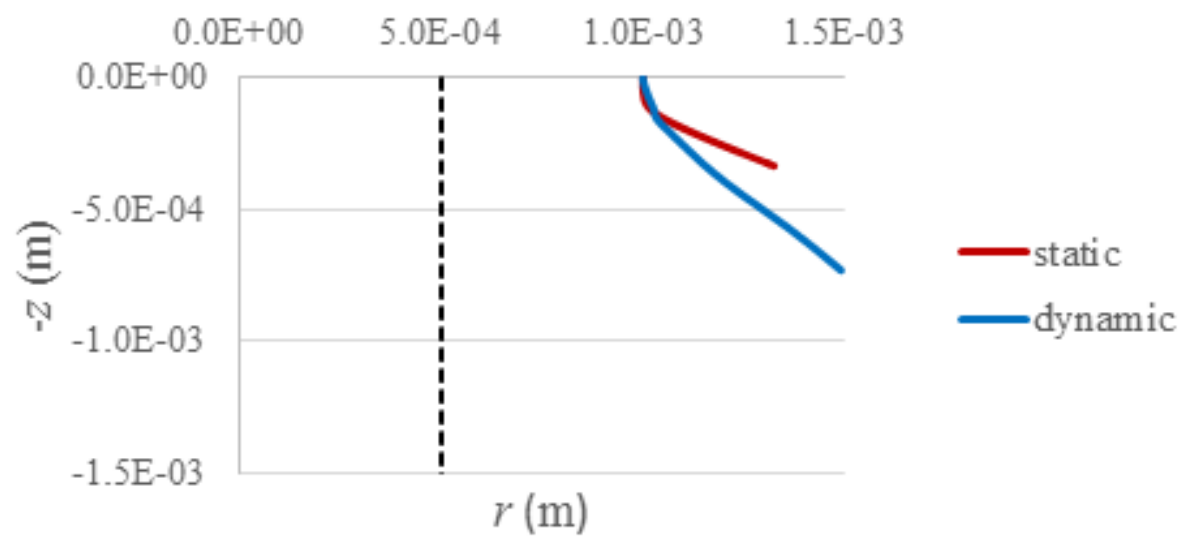


Figure 15: Direct comparison of cone cracks created from an existing flaw, one millimeter from the center of a cylindrical flat-punch indenter, for both quasi-static ( $\left.c_{d} \tau / a=1.14 \times 10^{6}\right)$ and dynamic $\left(c_{d} \tau / a=1.14\right)$ loading cases. The indenter radius was taken to be $a=500 \mu \mathrm{m}$ and the dilatational wave speed $c_{d}=5700 \mathrm{~m} / \mathrm{s}$. The Poisson

421 The wave speed $c_{d}=5700 \mathrm{~m} / \mathrm{s}$ and Poisson ratio $v=0.21$ are typical of soda-lime glass. One

422 observation is that as the radius of the initial flaw $\left(r_{0}\right)$ increases the length of the crack in the 423 quasi-static case decreases more rapidly than in the dynamic case. It can also be seen that the 424 initial portion of the trajectory, in the quasi-static case, tends to be perpendicular to the surface 425 before turning sharply; this does not seem to happen in the dynamic case. Figure 15 illustrates 426 the difference in shape and crack angle for growth under quasi-static and wave fields. In the case 427 of wave fields the crack grows at a significantly larger angle, with respect to the surface, than in 428 the quasi-static case.

429 Figures 16 and 17 demonstrate how dramatic the difference is between the quasi-static and 430 dynamic cases when it comes to how quickly the crack length decays with the distance between 431 flaw location and the edge of the indenter. 


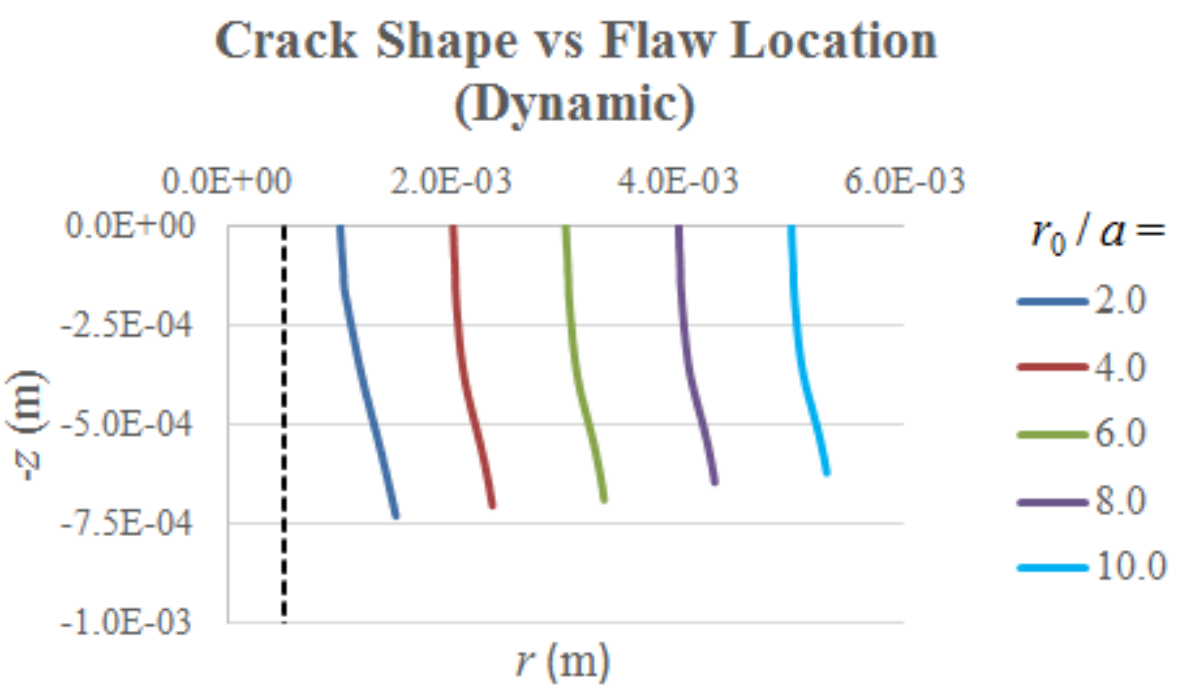

433

Figure 16: Cone cracks created from existing flaws, at relatively large distances from the edge of a cylindrical flat-punch indenter, by a dynamic load of strength $F_{0}=20 \mathrm{~N}$. The indenter radius was taken to be $a=500 \mu \mathrm{m}$, the ramp time $\tau=10^{-7} \mathrm{~s}$, and the dilatational wave speed $c_{d}=5700 \mathrm{~m} / \mathrm{s}\left(c_{d} \tau / a=1.14\right)$. The Poisson ratio was assumed to be $v=0.21$ which is typical of soda-lime glass. It is striking how slowly the crack length falls off with distance from the edge of the indenter. 


\section{Crack Length vs Relative Flaw Location}

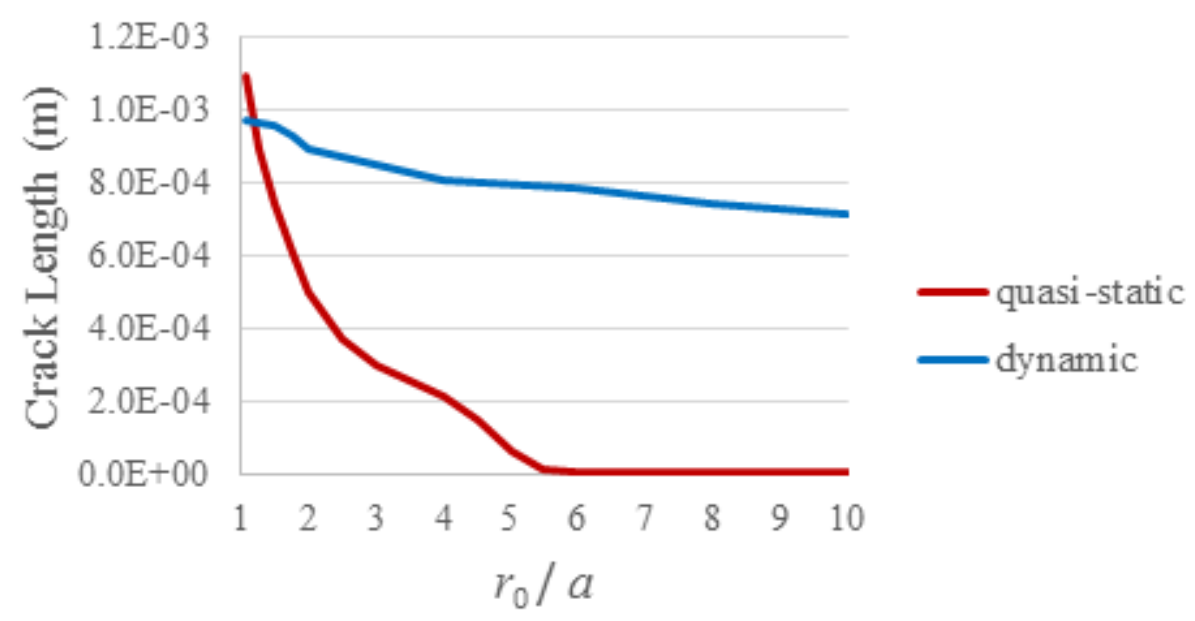

443

444

445

446

453 Figure 17 clearly shows that in the quasi-static case the crack length is essentially zero for $454 \quad r_{0} / a>5$ while in the dynamic case the crack length has only decreased by about $25 \%$ for 455

Figure 17: Comparison of crack length versus initial flaw location for both quasi-static $\left(c_{d} \tau / a=1.14 \times 10^{6}\right)$ and dynamic $\left(c_{d} \tau / a=1.14\right)$ loading cases for a cylindrical flatpunch indenter with a total load strength $F_{0}=20 \mathrm{~N}$. The indenter radius was $a=500 \mu \mathrm{m}$ and the dilatational wave speed $c_{d}=5700 \mathrm{~m} / \mathrm{s}$. The Poisson ratio was assumed to be $v=0.21$ which is typical of soda-lime glass. It is striking how in the quasi-static case the cracks become negligibly small above $r_{0} \approx 5 a$ while in the dynamic case they have only decreased by about $25 \%$ at $r_{0}=10 a$.

$r_{0} / a \approx 10$

The reason for the slow decay in the dynamic case is demonstrated in Figure 18. For dynamic crack growth the crack length is essentially determined by the amount of time the stress intensity factor stays above the threshold value for saturation of the crack speed at $1500 \mathrm{~m} / \mathrm{s}$ 
$459\left(K_{I} \approx 9 \times 10^{5} \mathrm{~Pa} \cdot \mathrm{m}^{1 / 2}\right)$. As can be seen in the figure this amount of time decreases only very

460 slowly as the distance between the flaw location and indenter edge increases.

\section{Intensity Factor vs Time}

(Dynamic)

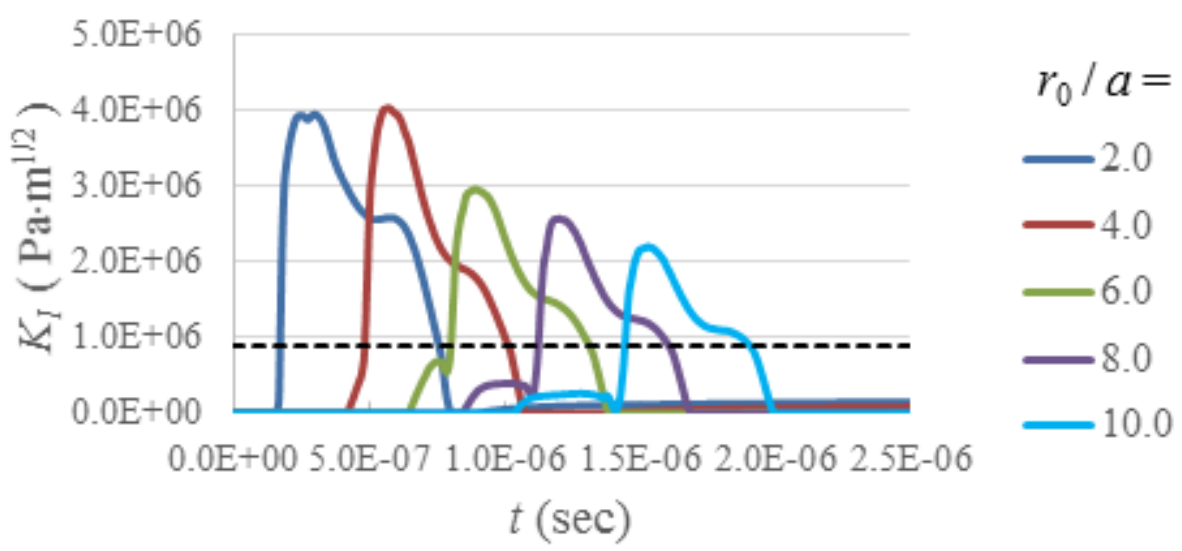

Figure 18: Intensity factor $K_{I}$ versus time for cone cracks initiating from flaws at various distances from the center of a cylindrical flat-punch indenter. The indenter has a radius $a=500 \mu \mathrm{m}$ and a total load $F_{0}=20 \mathrm{~N}$ with ramp time $\tau=10^{-7} \mathrm{~s}$. The dilatational wave speed and Poisson ratio were assumed to be $c_{d}=5700 \mathrm{~m} / \mathrm{s}$ and $v=0.21$ respectively which are typical values for soda-lime glass. Since we are dealing

471 Another striking difference between the quasi-static and dynamic cases is illustrated in figures

472 19-22. It was pointed out previously $[17,31]$ that in the quasi-static case the crack angle is

473 strongly dependent on the Poisson ratio $v$. This is demonstrated in Figure 19. Interestingly this

474 strong dependence is not found in the dynamic case as shown in Figure 20. Perhaps even more

475 dramatic is the difference in how the Poisson ratio affects the crack length in the two cases. As 
476 shown in Figure 21 the quasi-static crack length decreases dramatically with increasing $v$ while

477 in the dynamic case, as shown in Figure 22, the length actually increases slowly with increasing $478 v$.

\section{Effect of Poisson Ratio on Crack Shape (Quasi-Static)}

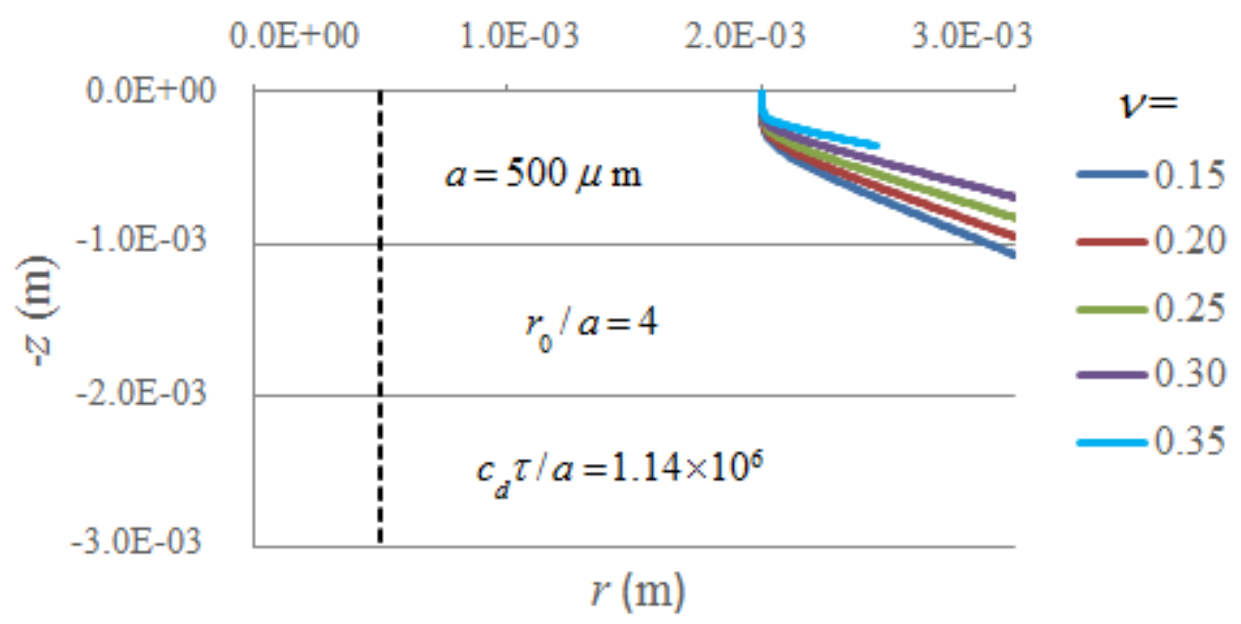

Figure 19: Cone cracks created from an existing flaw by a cylindrical flat-punch indenter of radius $a=500 \mu \mathrm{m}$ and total load $F_{0}=200 \mathrm{~N}$ with ramp rate $\tau=0.1 \mathrm{~s}$. The dilatational wave speed was assumed to be $c_{d}=5700 \mathrm{~m} / \mathrm{s}$. The initial flaw location was $r_{0}=4 a$. The different curves are for different values of the Poisson ratio $v$. In this quasi-static regime $\left(c_{d} \tau / a=1.14 \times 10^{6}\right)$ the angle that the crack grows at, with respect to the surface, is strongly dependent on the value of the Poisson ratio. 


\section{Effect of Poisson Ratio on Crack Shape (Dynamic)}

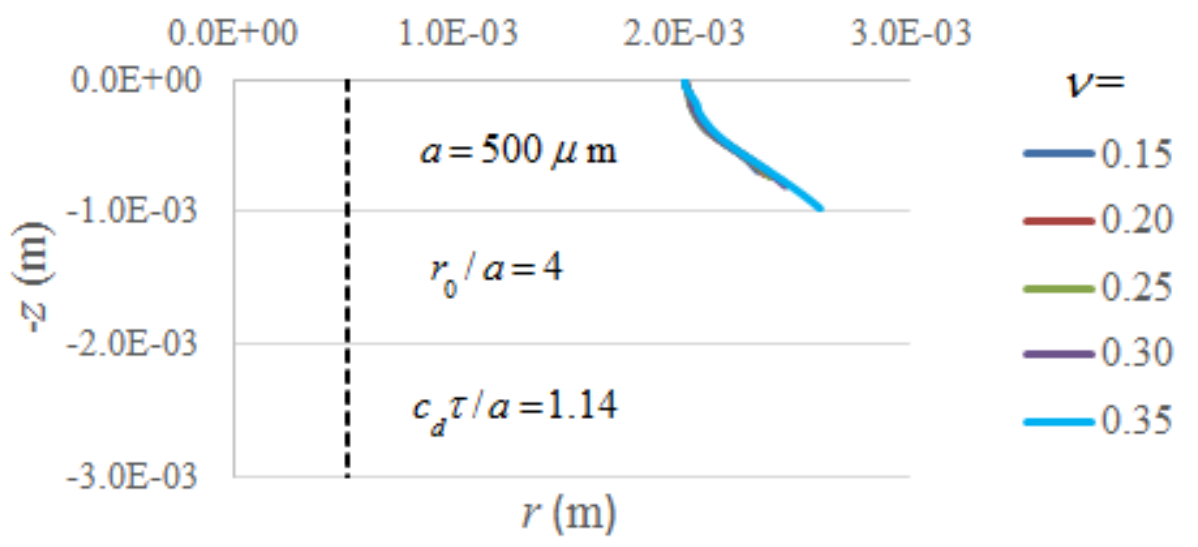

Figure 20: Cone cracks created from an existing flaw by a cylindrical flat-punch indenter of radius $a=500 \mu \mathrm{m}$ and total load $F_{0}=20 \mathrm{~N}$ with ramp rate $\tau=10^{-7} \mathrm{~s}$. The dilatational wave speed was assumed to be $c_{d}=5700 \mathrm{~m} / \mathrm{s}$. The initial flaw location was $r_{0}=4 a$. The different curves are for different values of the Poisson ratio $v$. In this dynamic regime $\left(c_{d} \tau / a=1.14\right)$ the angle that the crack grows at, with respect to the surface, is essentially independent on the value of the Poisson ratio. This is completely different behavior compared to the quasi-static case. 


\section{Effect of Poisson Ratio on Crack Length (Quasi-static)}

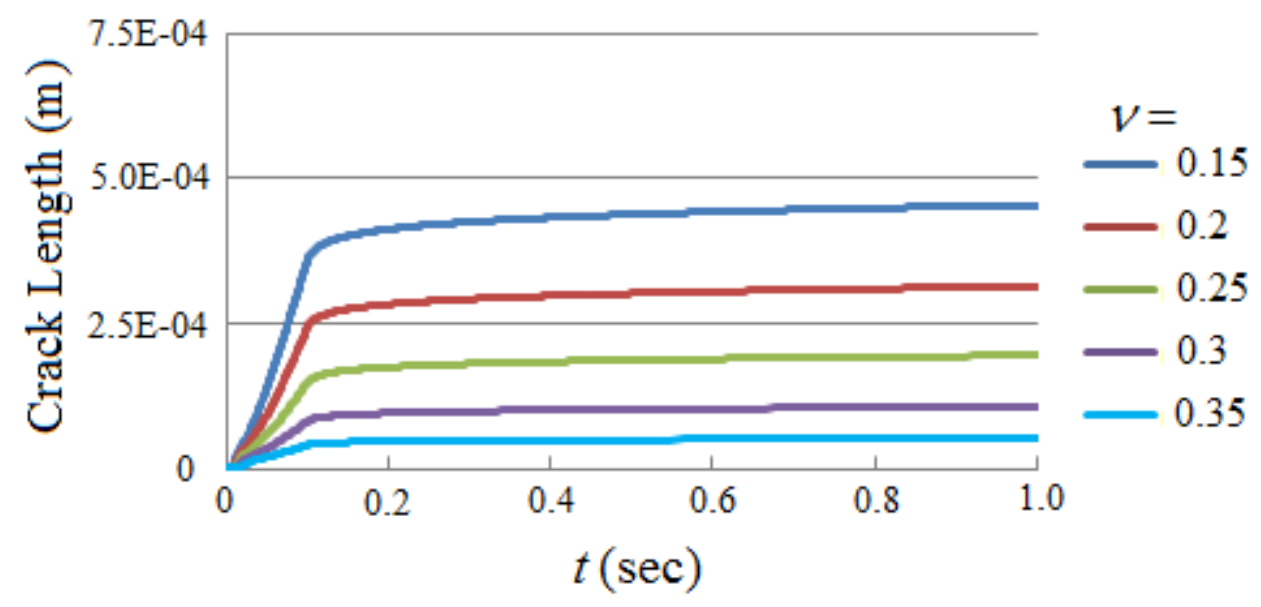

Figure 21: Crack length versus time for cone cracks generated from an existing flaw by a cylindrical flat-punch indenter of radius $a=500 \mu \mathrm{m}$ and total load $F_{0}=200 \mathrm{~N}$ with ramp rate $\tau=0.1 \mathrm{~s}$. The dilatational wave speed was assumed to be $c_{d}=5700 \mathrm{~m} / \mathrm{s}$. The initial flaw location was $r_{0}=4 a$. The different curves are for different values of the Poisson ratio $v$. It can be seen that, for this quasi-static case $\left(c_{d} \tau / a=1.14 \times 10^{6}\right)$, as $v$ increases the final crack length rapidly decreases. 


\section{Effect of Poisson Ratio on Crack Length (Dynamic)}

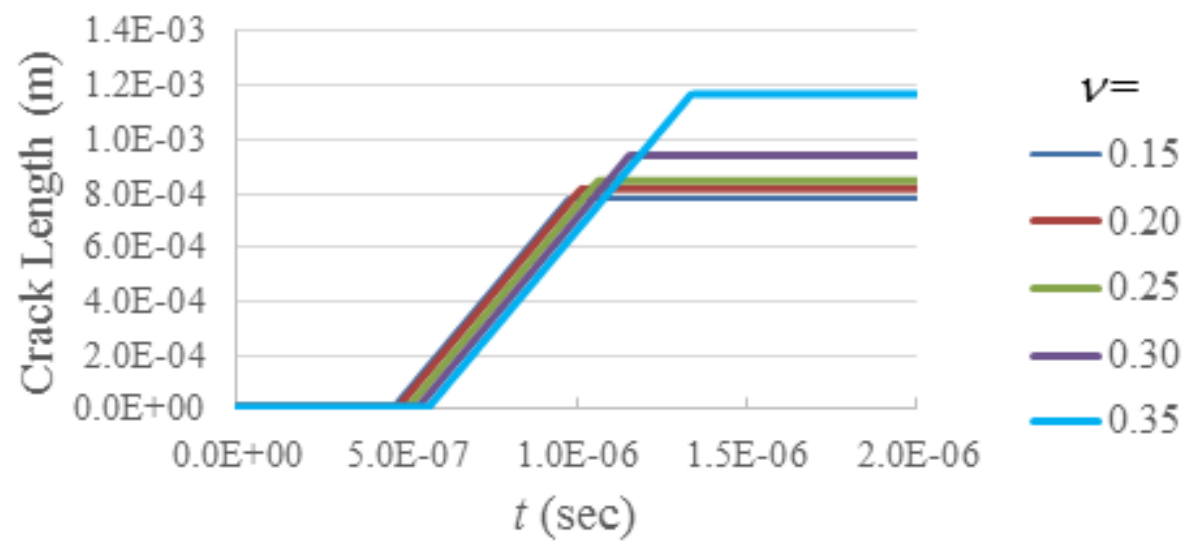

Figure 22: Crack length versus time for cone cracks generated from an existing flaw by a cylindrical flat-punch indenter of radius $a=500 \mu \mathrm{m}$ and total load $F_{0}=20 \mathrm{~N}$ with ramp rate $\tau=10^{-7} \mathrm{~s}$. The dilatational wave speed was assumed to be $c_{d}=5700 \mathrm{~m} / \mathrm{s}$. The initial flaw location was $r_{0}=4 a$. The different curves are for different values of the Poisson ratio $v$. It can be seen that, for this dynamic case $\left(c_{d} \tau / a=1.14\right)$, as $v$ increases the final crack length slowly increases. This behavior is drastically different than what is found for the quasi-static case.

\section{5. Conclusions}

514 In the present work a computational scheme to study surface wave effects was implemented.

515 Use of the Laplace transform enabled analytical expressions for the various quantities of interest

516 to be obtained in the transformed space. Numerical inversion of these closed-form expressions

517 provides stress and displacement distributions as functions of space and time. The method was

518 first compared to the known analytical solutions for the surface displacement due to the idealized

519 case of a suddenly-applied point load. It was next applied to the problem of cone crack

520 extension from an existing flaw under dynamic loading of a frictionless cylindrical punch. The 
521 computed cone cracks under dynamic loading conditions showed significant differences

522 compared to the case of quasi-static loading conditions.

523 It is possible to extend this scheme to study the effects of indenter elasticity, interface friction,

524 and surface coatings. Including the effects of indenter properties and friction would provide

525 insight into whether the initial stage of failure under dynamic loading is strongly influenced by

526 indenter or projectile type as it is in the static case. The effects of coating properties, layer

527 thickness, and degree of bonding at the interface could also be studied to yield insights into the

528 role of surface constraints in improving ballistic response of ceramics. The incorporation of the

529 above mentioned effects into the method presented below will be the subject of a future

530 publication.

\section{Acknowledgements}

533 The research reported in this document was performed in connection with contract/instrument

534 W911QX-14-C-0016 with the U.S. Army Research Laboratory. The views and conclusions

535 contained in this document are those of TKC Global and the U.S. Army Research Laboratory.

536 Citation of manufacturer's or trade names does not constitute an official endorsement or

537 approval of the use thereof. The U.S. Government is authorized to reproduce and distribute 538 reprints for Government purposes notwithstanding any copyright notation hereon.

542 [1] S. Sarva, S. Nemat-Nasser, J. McGee, J. Issacs, Int. J. Imp. Eng. 34, pp. 277-302 (2007).

543 [2] A. Samiee, J. Issacs, S. Nemat-Nasser, Proceedings of SPIE 7644 (2010). 
544 [3] P. R. S. Reddy, V. Madhu, K. Ramanjaneyulu, T. Balakrishna Bhat, K. Jayaraman, and N. K.

545 Gupta, Def. Sci. J. 58, pp. 264-274 (2008).

546 [4] J. McDonald and S. Satapathy, unpublished.

547 [5] Lord Rayleigh, Proc. London Math. Soc. 17, pp. 4-11 (1885).

548 [6] A. E. H. Love, Some Problems of Geodynamics, Cambridge University Press, London 549 (1911).

550 [7] R. Stoneley, Proc. Roy. Soc. A 106, pp. 416-428 (1924).

551 [8] K. Sezawa and K. Kanai, Bull. Earthquake Res. Inst.17, pp. 1-8 (1939).

552 [9] J. G. Scholte, Monthly Notices Roy. Astr. Soc.: Geophys. Suppl.5, pp. 120-126 (1947).

553 [10] B. A. Bolt and J. C. Butcher, Aus. J. Phys. 13, pp. 498-503 (1960).

554 [11] K. Sezawa and K. Kanai, Bull. Earthquake Res. Inst. 12, pp. 251-257 (1935).

555 [12] K. Sezawa and K. Kanai, Bull. Earthquake Res. Inst. 15, pp. 845-849 (1937).

556 [13] F. J. Serón, F. J. Sanz, M. Kindelán, and J. I. Badal, Comm. Appl. Num. Meth. 6, pp. 359$557368(1990)$

558 [14] Y. M. Tsai, Int. J. Solids Structures 7, pp. 543-558 (1971).

559 [15] G. Subhash, S. Maiti, Philippe H. Geubelle, and D. Ghosh, J. Am. Ceram. Soc. 91, pp. 27775602791.

561 [16] B. Schuster, unpublished. 
562 [17] B. R. Lawn, T. R. Wilshaw, and N. E. W. Hartley, Int. J. Frac. 10, pp. 1-16 (1974).

563 [18] C. Chao, J. Appl. Mech. 27, pp. 559-567 (1960).

564 [19] K. F. Graff, Wave Motion in Elastic Solids, Dover Publications, New York (1991).

565 [20] H. G. Georgiadis, D. Vamvatsikos, I. Vardoulakis, Comp. Mech. 24, pp. 90-99 (1999).

566 [21] P. Wynn, Math Tables Aids Comp. 10, pp. 91-96 (1956).

567 [22] R. Piessens, E. de Docncker-Kapenga, C. W. Überhuber, D. K. Kahaner, Quadpack: A

568 Subroutine Package for Automatic Integration, Springer-Verlog, Berlin Heidelberg, Germany 569 (1983).

570 [23] W. H Press, S. A. Teukolsky, W. T. Vetterling, B. P. Flannery, Numerical Recipes in

571 Fortran $2^{\text {nd }}$ Edition, Cambridge University Press, Cambridge (1992).

572 [24] L. D’Amore, G. Laccetti, and A. Murli, ACM Trans. Math. Soft. 25, pp. 279-305 (1999).

573 [25] C. L. Pekeris, Geophysics 41, pp. 469-480 (1955).

574 [26] A. C. Fischer-Cripps, Introduction to Contact Mechanics $2^{\text {nd }}$ Edition, Springer, New York 575 (2007).

576 [27] Y. M. Tsai and H. Kolsky, J. Mech. Phys. Solids 15, pp. 263-278 (1967).

577 [28] H. Hertz, Hertz's Miscellaneous Papers, Macmillan, London, U. K. (1896).

578 [29] L. B. Freund, Dynamic Fracture Mechanics, Cambridge University Press, Cambridge, UK 579 (1990).

580 [30] K. Ravi-Chander, Dynamic Fracture, Elsevier, Oxford, UK (2004). 
[31] C. Kocer and R. E. Collins, J. Am. Ceram. Soc. 81, pp. 1736-1742 (1998).

582

583

584

585

586

587

588

589

590

591

592

593

594

Inserting this expression along with the expressions in equation (7) into equation (8) gives:

\section{Appendix (Coefficients for various spatial distributions of the loading function):}

\section{Delta-Function Load:}

For a point-source (delta function) load, with strength $F_{0}$, the expression for $F(r)$ in equation

(8) becomes:

$$
F(r)=\frac{F_{0}}{2 \pi} \frac{\delta(r)}{r}=\frac{F_{0}}{2 \pi} \int_{0}^{\infty} k J_{0}(k r) d k
$$

$$
A(k, s)=-\frac{F_{0}}{2 \pi \mu} \frac{k\left(\kappa_{s}^{2}+k^{2}\right)}{\left(2 k^{2}+k_{s}^{2}\right)^{2}-4 \kappa_{d} \kappa_{s} k^{2}} \bar{G}(s)
$$

$$
B(k, s)=-\frac{F_{0}}{2 \pi \mu} \frac{2 \kappa_{d} k}{\left(2 k^{2}+k_{s}^{2}\right)^{2}-4 \kappa_{d} \kappa_{s} k^{2}} \bar{G}(s)
$$

\section{Gaussian Load:}

597 For a load of strength $F_{0}$ distributed as a Gaussian pulse of width $a$, the expression for $F(r)$ is:

$$
F(r)=\frac{F_{0}}{\pi a^{2}} \exp \left(-\frac{r^{2}}{a^{2}}\right)=\frac{F_{0}}{2 \pi} \int_{0}^{\infty} k \exp \left(-\frac{k^{2} a^{2}}{4}\right) J_{0}(k r) d k
$$

601 This gives the following expressions for the coefficients: 
603

$$
A(k, s)=-\frac{F_{0}}{2 \pi \mu} \frac{k\left(\kappa_{s}^{2}+k^{2}\right)}{\left(2 k^{2}+k_{s}^{2}\right)^{2}-4 \kappa_{d} \kappa_{s} k^{2}} \exp \left(-\frac{k^{2} a^{2}}{4}\right) \bar{G}(s)
$$

$$
B(k, s)=-\frac{F_{0}}{2 \pi \mu} \frac{2 \kappa_{d} k}{\left(2 k^{2}+k_{s}^{2}\right)^{2}-4 \kappa_{d} \kappa_{s} k^{2}} \exp \left(-\frac{k^{2} a^{2}}{4}\right) \bar{G}(s)
$$

604

605 It is clear from the above expressions that the coefficients in (A-4) reduce to those in (A-2) as

$606 \quad a \rightarrow 0$

607

608 Cylindrical Punch:

609 For a load of strength $F_{0}$ distributed over a cylindrical flat-punch indenter of radius $a$, the

610 expression for $F(r)$ is:

611

612

$$
F(r)=\left\{\begin{array}{cc}
\frac{F_{0}}{2 \pi a^{2}}\left(1-\frac{r^{2}}{a^{2}}\right)^{-1 / 2}=\frac{F_{0}}{2 \pi a} \int_{0}^{\infty} \sin (k a) J_{0}(k r) d k & r<a \\
0 & r>a
\end{array}\right.
$$

613 which gives:

614

$$
A(k, s)=-\frac{F_{0}}{2 \pi \mu} \frac{k\left(\kappa_{s}^{2}+k^{2}\right)}{\left(2 k^{2}+k_{s}^{2}\right)^{2}-4 \kappa_{d} \kappa_{s} k^{2}} \frac{\sin (k a)}{k a} \bar{G}(s)
$$

$$
B(k, s)=-\frac{F_{0}}{2 \pi \mu} \frac{2 \kappa_{d} k}{\left(2 k^{2}+k_{s}^{2}\right)^{2}-4 \kappa_{d} \kappa_{s} k^{2}} \frac{\sin (k a)}{k a} \bar{G}(s)
$$

615 


\section{Constant load over circular area:}

617 For a load of strength $F_{0}$ distributed uniformly over a circular area of radius $a$, the expression 618 for $F(r)$ is:

619

620

$$
F(r)=\left\{\begin{array}{cc}
\frac{F_{0}}{\pi a^{2}}=\frac{F_{0}}{\pi a} \int_{0}^{\infty} J_{1}(k a) J_{0}(k r) d k & r<a \\
0 & r>a
\end{array}\right.
$$

621 which gives:

622

$$
\begin{aligned}
& A(k, s)=-\frac{F_{0}}{\pi \mu} \frac{k\left(\kappa_{s}^{2}+k^{2}\right)}{\left(2 k^{2}+k_{s}^{2}\right)^{2}-4 \kappa_{d} \kappa_{s} k^{2}} \frac{J_{1}(k a)}{k a} \bar{G}(s) \\
& B(k, s)=-\frac{F_{0}}{\pi \mu} \frac{2 \kappa_{d} k}{\left(2 k^{2}+k_{s}^{2}\right)^{2}-4 \kappa_{d} \kappa_{s} k^{2}} \frac{J_{1}(k a)}{k a} \bar{G}(s)
\end{aligned}
$$

